\title{
Involvement of Nucleotide-Binding Oligomerization Domain-Like Receptor Family Pyrin Domain Containing 3 Inflammasome in the Pathogenesis of Liver Diseases
}

OPEN ACCESS

Edited by:

Yang Zhang,

University of Houston, United States

Reviewed by:

Mi Wang,

Huazhong University of Science and Technology, China

Mariateresa Giuliano,

University of Campania Luigi Vanvitelli,

Italy

*Correspondence:

Hongqin Yang

hqyang@fjnu.edu.cn

Zhenghong Zhang

zhangzh@fjnu.edu.cn

Specialty section:

This article was submitted to

Molecular Medicine

a section of the journal

Frontiers in Cell and Developmental

Biology

Received: 03 December 2019

Accepted: 19 February 2020

Published: 10 March 2020

Citation:

Shi C, Yang H and Zhang Z (2020) Involvement of Nucleotide-Binding

Oligomerization Domain-Like

Receptor Family Pyrin Domain

Containing 3 Inflammasome

in the Pathogenesis of Liver Diseases.

Front. Cell Dev. Biol. 8:139.

doi: 10.3389/fcell.2020.00139

\section{Congjian Shi, Hongqin Yang* and Zhenghong Zhang*}

Provincial Key Laboratory for Developmental Biology and Neurosciences, Key Laboratory of Optoelectronic Science and Technology for Medicine of Ministry of Education, College of Life Sciences, Fujian Normal University, Fuzhou, China

The inflammasome is widely acknowledged for its crucial role in the pathogenesis of cancers and many neurodegenerative, metabolic, and auto-inflammatory diseases in recent years. Multiple types of inflammasomes exist. However, nucleotide-binding oligomerization domain-like receptor family pyrin domain containing 3 (NLRP3) inflammasome is the most often investigated inflammasome and has come to limelight in recent studies. NLRP3 inflammasome is a multi-protein complex. Its activation can cause the cleavage of inactive pro-caspase- 1 into activated caspase-1, that ultimately promotes the transformation of pro-interleukin (IL)-1 $\beta$ and pro-IL-18 into biologically-active $\mathrm{IL}-1 \beta$ and $\mathrm{IL}-18$, respectively. These processes lead to the local inflammatory responses and induce pyroptosis, causing disparaging effects. Recently, numerous studies have shown that NLRP3 inflammasome plays an important role in the pathogenesis of liver diseases, including non-alcoholic fatty liver disease, liver fibrosis, cirrhosis, and hepatocellular carcinoma. Liver diseases have become a severe health burden worldwide, and there is adequate evidence indicating that the regulation of NLRP3 inflammasome acts as a guard against hazard to liver. In this review, we provide a straightforward overview of NLRP3 inflammasome as well as several frequent liver diseases. We then discuss the contribution and regulation of NLRP3 inflammasome during the pathogenesis of liver diseases, which may provide an important indication for the prevention and treatment of various liver diseases.

Keywords: NLRP3 inflammasome, inflammation, non-alcoholic fatty liver disease, liver fibrosis, cirrhosis, hepatocellular carcinoma

\section{INTRODUCTION}

Currently, living conditions have greatly improved as compared to the earlier times. Humans consume considerably amount of nutrition, alcohol, and carbohydrate that upon combining with other endogenous and exogenous influential factors, aggravate liver burden ultimately leading to the lesions of liver. Liver plays an irreplaceable role in body metabolism, and liver diseases seriously affect normal human life. Common liver diseases including non-alcoholic fatty liver disease (NAFLD), liver fibrosis, cirrhosis, and hepatocellular carcinoma (HCC) are serious health threats to the people worldwide (Bouattour et al., 2018; Schuppan et al., 2018; Vonghia et al., 2019). 
Patients in the early stage of liver diseases usually have mild symptoms, which are often not felt by the patients. Thus, liver diseases are greatly concealed health dangers for humans worldwide. Paying attention to the pathogenesis of liver diseases and searching for treatments that are more efficient is thus, of utmost importance.

NLRP3 inflammasome has attracted considerable attention during recent years. It is basically a kind of multimeric protein complex which consists of NLRP3, apoptosis-associated specklike protein containing a caspase activation and recruitment domain (ASC) and pro-caspase-1 (Chen et al., 2016; Wang et al., 2016, 2019). Latest studies indicate that the activity of NLRP3 inflammasome is closely related with diversified diseases, including rheumatoid arthritis (Wu et al., 2019), breast cancer (Ershaid et al., 2019), pleurisy (Yang S. et al., 2019), systemic lupus erythematosus (Tan et al., 2019), cardiovascular disease (Zhang Y. et al., 2019), and renal fibrosis (Guo et al., 2017). Till date, NLRP3 inflammasome is widely studied in liver diseases with its activity in liver diseases being comprehensively investigated, and numerous studies showing a key role of NLRP3 inflammasome in the pathogenesis of liver diseases, especially NAFLD, liver fibrosis, cirrhosis, and HCC (Wu et al., 2015; Yang et al., 2016; Wei et al., 2019). Consequently, there is a critical need to understand the effect of NLRP3 inflammasome in various kinds of liver diseases mentioned above that may be helpful in developing potential treatments to regain normal liver functions.

\section{NLRP3 INFLAMMASOME}

The inflammasome is one of the most important multimeric protein complexes that participate in immune system functions, and is widely known as an intracellular inflammatory machinery (Boini et al., 2014; Chen et al., 2015). It plays a pivotal role in recognizing infection, repairing damaged tissue and initiating the process of pathogen clearance (Strowig et al., 2012). The inflammasome is assembled by intra-cytoplasmic sensor protein called pattern recognition receptors (PRRs), adaptor protein ASC, and effector protein pro-caspase-1 (Fan et al., 2019). The types of PRRs present in it, which define the designation of inflammasome. The PRRs can perceive the pathogen-associated molecular patterns (PAMPs) and dangerassociated molecular patterns (DAMPs), and then generate immune responses to eliminate infections and repair injured tissues (Bortolotti et al., 2018).

Among multiple types of inflammasomes, NLRP3 inflammasome has received extra attention, with definitive studies on its function and structure. NLRP3 inflammasome is critical for immune defense, which can withstand bacterial, fungal, and viral infections (Man and Kanneganti, 2015). Cause the activation of NLRP3 inflammasome is an inflammatory process, it must be strictly regulated. In recent studies, a two-step mechanism has been proposed to explain the process (Swanson et al., 2019). The mechanism includes the priming step and the activation step (Figure 1). During the priming step, endogenous cytokines or microbial components in cell membrane stimulate toll-like receptor (TLR), nucleotide-binding oligomerization domain (NOD) 2 or tumor necrosis factor (TNF) receptor leading to the activation of nuclear factor $-\kappa B(N F-\kappa B)$. NF- $\kappa B$ then promotes the synthesis of NLRP3 and the expression of pro-IL-1 $\beta$ and pro-IL-18 (Guo et al., 2016; Mathur et al., 2018). During the activation step, stimulation like $\mathrm{K}^{+}$efflux, $\mathrm{Ca}^{2+}$ mobilization, $\mathrm{Na}^{+}$influx, chloride efflux, reactive oxygen species (ROS), mitochondrial dysfunction, and lysosomal damage generate activation signals, leading to the assembly of NLRP3 inflammasome with caspase-1 mediating IL- $1 \beta$ and IL-18 excretion (Zhang et al., 2015; Kelley et al., 2019). IL-1 $\beta$, primarily excreted by mononuclear macrophage cells, is an effective pro-inflammatory cytokine that recruits innate immune cells to the site of infection and regulates acquired immune cells. IL-18 stimulates the production of interferon- $\gamma$ (IFN- $\gamma$ ) and enhances the ability of T cells and natural killer (NK) cells (van de Veerdonk et al., 2011). What's more, capase-1 makes it possible for the non-conventional secretion of numerous cytosolic proteins (Mangan et al., 2018). In this process, activated caspase- 1 also dissociates gasdermin D (GSDMD), and regulates the N-terminal domain of GSDMD by forming pores in the plasma membrane. It thus, stimulates an inflammatory form of cell death termed pyroptosis (An et al., 2019), which is persistently observed during microbial infections that combine the features of apoptosis and necrosis.

Accumulated evidences have already demonstrated that NLRP3 inflammasome participates in the pathogenesis of liver diseases like NAFLD, liver fibrosis, cirrhosis, and HCC. Plentiful existing literatures summarized the rough relationship between NLRP3 inflammasome and liver diseases, involved the changes of NLRP3 inflammasome components expression under different liver disease states, and knocked out the genes that play pivotal roles in the assembly process of NLRP3 inflammasome to observe the occurrence and development of liver diseases. However, a few literatures have pointed out the specific mechanism of NLRP3 inflammasome influencing the pathogenesis of liver diseases, more studies should be conducted to explore the definite underlying mechanism. And further studies focused on NLRP3 inflammasome activity may provide novel strategies for the treatments of these liver diseases.

\section{NLRP3 INFLAMMASOME AND NAFLD}

\section{NAFLD Pathogenesis}

NAFLD is one of the most common chronic liver diseases globally, which ranges from simple steatosis to steatohepatitis [non-alcoholic steatohepatitis (NASH)]. NAFLD is generally associated with insulin resistance, obesity, type 2 diabetes, and hyperlipidemia (Wan et al., 2016a). Approximately 20 30\% of people around the world are affected by NAFLD and its complications including a proportion of up to $75-100 \%$ obese people (Henao-Mejia et al., 2012). NAFLD is usually unnoticed by patients for its mild symptoms, therefore they are likely to neglect it (Shi et al., 2019). A "two-hit" hypothesis had been proposed to explain the pathogenesis of NAFLD. In the first hit, insulin resistance and lipid metabolism disorders lead to the excessive lipid deposition and increasing triglyceride (TG) 


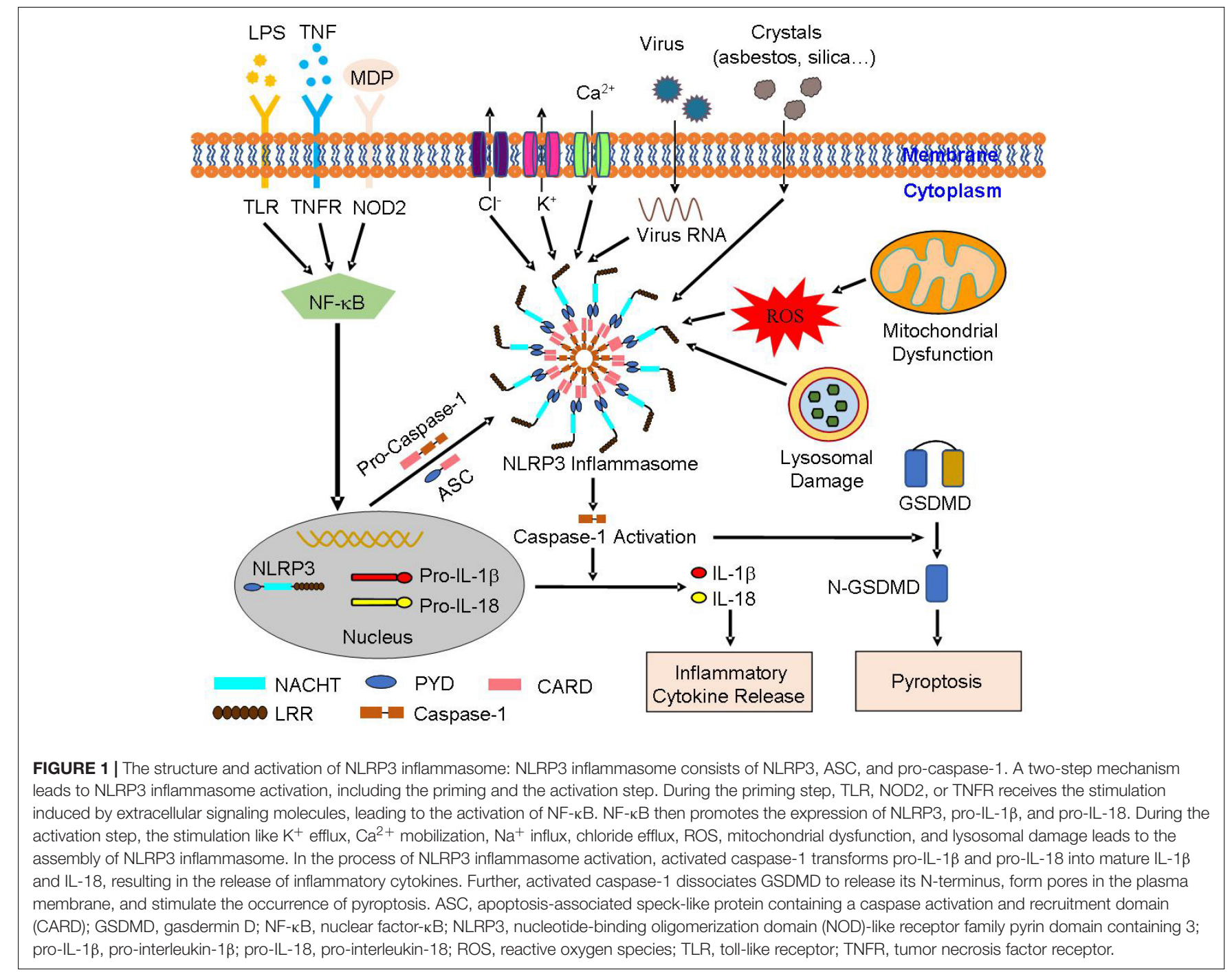

in hepatocytes. This is followed by the second hit, which is a multifactorial process including mitochondrial dysfunction, lipid peroxidation, and excess production of ROS via oxidative stress (Zhu et al., 2018). These events thus, cause liver damage and without effective control measures, the damage may progress to grave diseases like liver fibrosis, cirrhosis, and even HCC. Despite extensive studies on NAFLD, there is still no approved pharmacological agent for treating it with people mainly adopting precautions such as weight-loss and healthy lifestyle to overcome the disease.

\section{NLRP3 Inflammasome in NAFLD}

Recent studies have demonstrated that there is an intimate connection between NLRP3 inflammasome and NAFLD, with NAFLD mice showing an evident increased expression of NLRP3, ASC, and caspase- 1 in comparison to the mice fed by normal diet (Lv et al., 2019), and a large proportion of mice NAFLD models are caused by high fat diets. An identical consequence was affirmed in HepG2 and L02 cells that showed that inhibition of NLRP3 activity decreases uric acid-induced lipid accumulation in both the cell lines (Wan et al., 2016a). Furthermore, the studies using $\mathrm{NLRP}^{-/-}, \mathrm{ASC}^{-/-}$, and caspase $-1^{-/-}$mice suggest that the pathogenesis of NAFLD is associated with the activation of NLRP3 inflammasome (Henao-Mejia et al., 2012). Moreover, TNF- $\alpha$ level are evidently higher in NAFLD mice than that in normal mice. Mitsuyoshi et al. (2017) have reported that liver NLRP3, IL-1 $\beta$, IL-18, and pro-caspase-1 mRNA levels are significantly increased in NAFLD patients than those in healthy people, suggesting that NLRP3 inflammasome activation may promote the pathological progression of NAFLD. Chitturi and Farrell have reported that insulin resistance is a pivotal pathogenic factor associated with NAFLD, while inflammatory factors including IL- $1 \beta$, IL- 18 , and NF- $\mathrm{BB}$ have a close connection with insulin resistance. Moreover, the activation of these inflammatory factors is regulated by the assembly of NLRP3 inflammasome (Chitturi and Farrell, 2001). Under NAFLD state, hepatocytes are subjected to excessive oxidative stress; hence, the generation of excess ROS is inevitable. ROS as an intracellular danger signal makes thioredoxin interacting protein (TXNIP) to dissociate from thioredoxin (TRX), thus 
triggering the activation of NLRP3 inflammasome. Studies also indicate that TXNIP upregulation leads to the secretion of IL$1 \beta$ and IL-18 (Zheng et al., 2018). In a NASH model, mice with IL- $1 \alpha$ and IL-1 $\beta$ gene knockout noticeably prevented the pathological process from steatosis to steatohepatitis, while IL$1 \beta$ increases TG level in hepatocytes and induces hepatocytes death by collaborating with TNF- $\alpha$ (Wan et al., 2016b). All these evidences imply that NLRP3 inflammasome plays an important role in NAFLD, but the definite underlying mechanism remain elusive. Our team has a decent foundation for investigating NLRP3 inflammasome (Zhang Z. et al., 2019), and the steatosis models of mice livers and L02 cells were successfully established in our previous study (Shi et al., 2019), we intend to further explore the important role of NLRP3 inflammasome in the pathological process of NAFLD and detect the potential mechanism.

AMP-activated protein kinase (AMPK) activation has been proved to alleviate NAFLD state. It has demonstrated that the activation of peroxisome proliferator-activated receptors- $\delta$ (PPAR- $\delta$ ), a kind of nuclear receptor protein, restrains the decline of AMPK expression and increases the phosphorylation of AMPK- $\alpha$ (Lee H.J. et al., 2015). Moreover, AMPK activation inhibits the expression of IL- $1 \beta$ and caspase-1, and suppresses ROS generation (Lee H.J. et al., 2015), and AMPK/ROS pathway is closely related with the activation of NLRP3 inflammasome. The activation of NF-кB-related signaling pathway also has an accelerating effect in the pathogenesis of NAFLD (Xiao et al., 2018), while NF- $\mathrm{B}$ engages in the priming step of NLRP3 inflammasome activation. Other studies have shown that endoplasmic reticulum (ER) stress can induce the activation of NLRP3 inflammasome (Han et al., 2018), ultimately leading to hepatocyte death and NAFLD (Figure 2). Studies that aim at understanding the regulation of NLRP3 inflammasome may provide new potential strategy for the treatment of NAFLD. Resveratrol, a polyphenol widely present in grapes, pomegranates, blueberries, and mulberries, is reported to ameliorate NAFLD by suppressing the activation of NLRP3 inflammasome (Yang and Lim, 2014). A natural product such as honey, which made by bees, can protect liver during the progression of NAFLD through restraining NLRP3 inflammasome activity (Xiao et al., 2016). There are many similar substances, such as sulforaphane, salvianolic acid A, MCC950, andrographolide, silybin, salidroside, etc. That can be assessed for their effects on NLRP3 inflammasome. Since NLRP3 inflammasome is crucial for the pathogenesis of NAFLD and the initiation of liver inflammatory responses, suppressing its endogenous activation may have a favorable effect in improving NAFLD.

\section{NLRP3 INFLAMMASOME AND LIVER FIBROSIS/CIRRHOSIS}

\section{Liver Fibrosis/Cirrhosis}

Liver fibrosis is a wound healing process that occurs in the liver. It usually results from sustained, chronic liver injury, including viral hepatitis, long-term drinking, autoimmune diseases, and

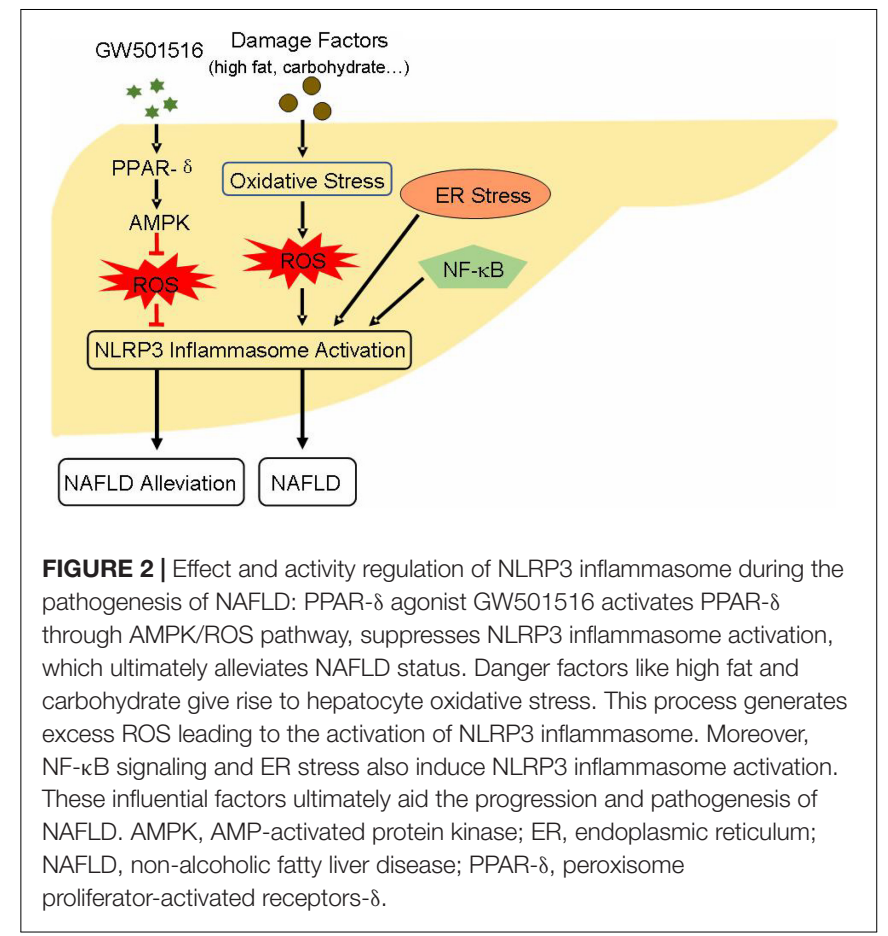

NAFLD (Seki and Brenner, 2015). In absence of intervention, liver fibrosis ultimately develops into cirrhosis with time and is characterized by extracellular matrix (ECM) substituting the bulk of liver parenchyma. The ECM can increase up to 10 -fold in cirrhosis compared with liver fibrosis (Schuppan et al., 2018), and cirrhosis is the final manifestation stage of liver fibrosis. For patients with advanced cirrhosis, liver transplantation is the only effective treatment. It is now widely accepted that the occurrence and development of liver fibrosis is tightly related with the activation of hepatic stellate cells (HSCs). HSCs in healthy liver can be detected between hepatocytes and sinusoidal endothelium. They are quiescent and function to store retinoids and generate glial fibrillary acidic protein (GFAP). However, sustained chronic liver injury causes the activation of HSCs and transforms them into myofibroblasts, while Kupffer cells are also activated in this process (Wang et al., 2018). Except HSCs, others cells with the capability of developing into myofibroblasts include endothelial cells, epithelial cells, biliary duct fibroblasts, mesenchymal cells, and mesangial cells (Artlett and Thacker, 2015). Myofibroblasts accumulate in fibrotic liver while they are rarely detected in healthy liver. Hence, they are considered as the principle effector cells of liver fibrosis. Moreover, myofibroblasts synthesize interstitial or fibrillar ECM, also referred to as scar tissue in liver parenchyma. The deposition of ECM is the morphological characteristic of liver fibrosis (Koyama et al., 2016). Chemokines, cytokines, growth factors, oxidative stress, and dominant ECM (mainly types I and III collagens) contribute in the pathogenesis of liver fibrosis (Sanchez-Valle et al., 2012). At present, the studies on the potential treatments of liver fibrosis primarily aim at preventing or reducing liver injury, transferring bone-marrow derived restorative macrophages or inhibiting inflammatory 
monocyte infiltration, promoting myofibroblasts apoptosis, and accelerating ECM degradation (Schuppan and Kim, 2013; Lee Y.A. et al., 2015).

\section{NLRP3 Inflammasome in Liver Fibrosis/Cirrhosis}

Accumulated evidences indicate that NLRP3 inflammasome plays an important role in the pathogenesis of liver fibrosis/cirrhosis. In the pathological process of liver fibrosis, the most discussed phenomenon is the activation of HSCs. The activation process is accompanied by increased levels of ECM, $\alpha$-smooth muscle actin ( $\alpha$-SMA), transforming growth factor- $\beta$ (TGF- $\beta$ ), platelet-derived growth factor (PDGF), and tissue inhibitor of metalloproteinase 1 (TIMP1) (Koyama et al., 2016). Studies have shown that wild type mice develop more severe liver fibrosis induced by carbon tetrachloride $\left(\mathrm{CCL}_{4}\right)$ as compared to the $\mathrm{NLRP}^{-/-}$and $\mathrm{ASC}^{-/-}$mice, as evidenced by higher $\alpha$-SMA expression and more Sirius Red staining area in the wild-type than in the knockout mice (Watanabe et al., 2009). Conversely, the activation of NLRP3 inflammasome in mice facilitates the pathological process of liver inflammation and fibrosis (Inzaugarat et al., 2019). Activated caspase-1, IL-18, and IL-1 $\beta$ signaling are also pivotal to the development of liver fibrosis resulting from the activation of NLRP3 inflammasome. Both IL- $1 \beta$ and IL-18 can induce the deposition of collagen (Artlett, 2012). MCC950, an agent that can block NLRP3 inflammasome, decreases the expression of liver caspase- 1 and the numbers of macrophages and neutrophils, ultimately alleviating liver fibrosis (Mridha et al., 2017). These studies provide overwhelming evidences about the association of NLRP3 inflammasome activation with accelerated liver fibrotic pathological process.

The renin-angiotensin system (RAS) possesses regulatory effects on liver fibrosis. Angiotensin II (Ang II), a key player in RAS, induces the generation of NADPH oxidase 4 (NOX4)/mitochondria-derived ROS and aggravates liver fibrosis, while NLRP3 inflammasome activation is tightly connect with NOX4/mitochondria-derived ROS (Cai et al., 2016). The inhibition of Ang II alleviates liver fibrosis by reducing the generation of Ang II-induced ROS and influencing NLRP3 inflammasome activation. In addition, Ang II stimulation induces ECM deposition and the proliferation of HSCs (Ning et al., 2017; You et al., 2019). Furthermore, crystalline monosodium urate is known to be an activator of NLRP3 inflammasome in vitro, that increases TGF- $\beta$ and collagen I expression in LX-2 cells (immortalized human HSCs) and primary mouse HSCs (Watanabe et al., 2009). NF-кB signaling is also important for the pathological process of liver fibrosis, while NLRP3 inflammasome activation is accompanied by the upregulation of NF- $\kappa \mathrm{B}$ signaling. Additionally, suppressing NF$\kappa \mathrm{B}$ signaling along with the promotion of HSCs apoptosis improves liver fibrosis (Zheng et al., 2019). These factors are intimately related to the activation of NLRP3 inflammasome, prospectively, it will be a splendiferous choice to prevent and treat liver fibrosis by probing the inhibition of NLRP3 inflammasome activity.

\section{NLRP3 INFLAMMASOME AND HCC}

\section{Hepatocellular Carcinoma}

Hepatocellular carcinoma is the most common liver malignancy, accounting for more than $90 \%$ of liver cancers, and a major cause of cancer-related mortality (Zhu et al., 2019). The situation of HCC is getting austere with its morbidity and mortality increasing year by year. Men are confronted with approximately threefold higher risk than women, on account of higher likelihood to smoking and drinking (El-Serag and Kanwal, 2014). Main risk factors of HCC are closely related to hepatitis $B$ virus (HBV), hepatitis C virus (HCV), alcoholic liver disease (ALD), NAFLD, liver fibrosis, obesity, diabetes, and smoking (Inchingolo et al., 2019). Most cases of HCC are detected at advanced stages and less than $40 \%$ of HCC patients have the chance to be treated surgically (Forner et al., 2012). Therefore, the diagnosis of HCC becomes particularly important. Current methods for evaluating HCC include histological observation, serum alpha-fetoprotein (AFP) level detection, computed tomography (CT), and magnetic resonance imaging (MRI) (Ayoub et al., 2019). The major clinical methods used in the treatment of HCC include surgical resection, liver transplantation, ablative therapies, chemoembolization, and systemic therapy (Akateh et al., 2019). Although many new therapeutic schedules are applied in clinical settings, the 5 -year survival rate is typically unfavorable in addition to adverse reactions and drug resistance associated with the use of anticancer drugs (Yang H. et al., 2019). Consequently, it is extremely necessary to find new targets for the treatment of HCC.

\section{NLRP3 Inflammasome in HCC}

As HCC is one of the foremost health hazards, increasing number of studies have been devoted for it. NLRP3 inflammasome has been demonstrated to play a significant role in NAFLD and liver fibrosis/cirrhosis. Hence, increasing attention has been endowed on understanding the regulation of NLRP3 inflammasome activity in the pathogenesis of HCC. However, the

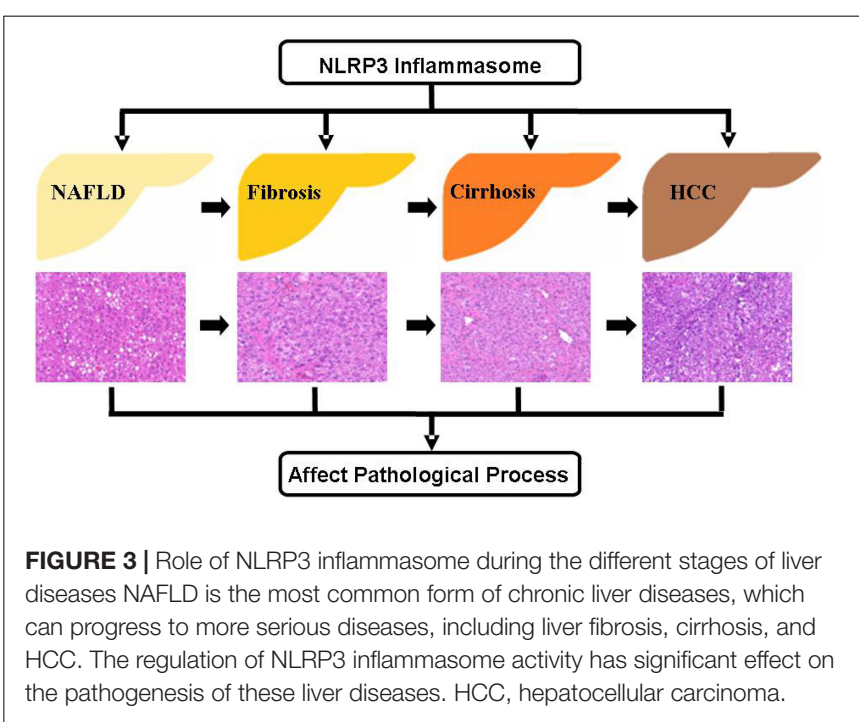


underlying mechanisms remain greatly controversial. Expression of NLRP3, ASC, caspase-1, and IL- $1 \beta$ are significantly decreased in HCC tissues as compared to the corresponding noncancerous liver tissues. Moreover, the expression of these genes also decreases progressively with the development of pathological and clinical stage of HCC (Wei et al., 2014). These results imply that the activation of NLRP3 inflammasome has protective effects in the pathogenesis of HCC. Taking into account the gender difference in the incidence of HCC, $17 \beta$-estradiol $\left(\mathrm{E}_{2}\right)$, and estrogen receptor (ER) $\beta$ have received extra consideration. Studies have shown that the expression of ER $\beta$ also decreases in the HCC tissues. Wei et al. (2015) have reported that HCC cell lines (SMMC7721, BEL7402, and HepG2 cells) treated with $\mathrm{E}_{2}$-activated NLRP3 inflammasome through $\mathrm{E}_{2} / \mathrm{ER} \beta / \mathrm{MAPK}$ signaling, eventually ameliorated HCC progression. Furthermore, the NLRP3 inflammasome activated by $\mathrm{E}_{2}$ treatment -induced caspase-1-dependent pyroptosis in HepG2 cells, led to the suppression of HCC progression (Wei et al., 2019).

However, there are contrary opinions about the influence of NLRP3 inflammasome activation in the pathogenesis of HCC. Luteoloside, a natural flavonoid with many pharmacological activities, suppresses the NLRP3 inflammasome activation and induces the inhibition of proliferation, invasion, and metastasis in HCC cells (Huh7 and SMMC7721) (Fan et al., 2014). Wan et al. (2018) have reported that microRNA-223-3p promotes apoptosis and inhibits the proliferation in Hep3B cells by suppressing the activation of NLRP3 inflammasome. These results suggest that NLRP3 inflammasome activation accelerates the pathological process of HCC. Thus, inhibiting the activity of NLRP3 inflammasome may become a novel potential target for treating HCC. The relationship between NLRP3 inflammasome and HCC has not been thoroughly studied yet, we need to make clear the definite molecular mechanism by which NLRP3 inflammasome affects the pathogenesis of HCC. Additional studies are essential to understand the relationship between NLRP3 inflammasome and HCC progression in future.

\section{SUMMARY}

Liver diseases have been considered as grave health hazards globally. Current studies have clearly shown that NAFLD has

\section{REFERENCES}

Akateh, C., Black, S. M., Conteh, L., Miller, E. D., Noonan, A., Elliott, E., et al. (2019). Neoadjuvant and adjuvant treatment strategies for hepatocellular carcinoma. World J. Gastroenterol. 25, 3704-3721. doi: 10.3748/wjg.v25.i28. 3704

An, N., Gao, Y., Si, Z., Zhang, H., Wang, L., Tian, C., et al. (2019). Regulatory mechanisms of the nlrp3 inflammasome, a novel immune-inflammatory marker in cardiovascular diseases. Front. Immunol. 10:1592. doi: 10.3389/ fimmu.2019.01592

Artlett, C. M. (2012). The role of the NLRP3 inflammasome in fibrosis. Open Rheumatol. J. 6, 80-86. doi: 10.2174/1874312901206010080

Artlett, C. M., and Thacker, J. D. (2015). Molecular activation of the NLRP3 Inflammasome in fibrosis: common threads linking divergent fibrogenic diseases. Antioxid. Redox. Signal. 22, 1162-1175. doi: 10.1089/ars.2014.6148 a high incidence in the population, and without effective interventions, it can progress toward liver fibrosis, cirrhosis, and eventually HCC (Figure 3). During the pathogenesis of NAFLD, liver fibrosis, and cirrhosis, the activation of NLRP3 inflammasome accelerates the pathological progression aggravating the symptoms of liver diseases on human body. In contrast, the impact seems to be reverse in HCC. Recent studies have shown that NLRP3 inflammasome activation acts as a defender in the pathogenesis of HCC. On the other hand, some other studies have indicated the reverse in case of HCC. Thus, it is worthwhile to explore more precisely the role of NLRP3 inflammasome in HCC as this area still has many controversies. Undoubtedly, there is no denying that NLRP3 inflammasome plays a critically function in the pathogenesis of liver diseases and further investigations aimed to delineate the effects of NLRP3 inflammasome in liver diseases are imperative. Fortunately, the discovery and exploration of NLRP3 inflammasome has provided a novel insight into the liver diseases, which may be further exploited as an important target for the prevention and treatment of liver diseases.

\section{AUTHOR CONTRIBUTIONS}

CS wrote the manuscript draft. CS, HY, and ZZ revised it. All authors read and approved the final version of the manuscript for publication.

\section{FUNDING}

This study was supported by National Key Basic Research Program of China (2015CB352006), National Natural Science Foundation of China (61335011 and 61405035), Program for Changjiang Scholars and Innovative Research Team in University (IRT-15R10), and Natural Science Foundation of Fujian Province (2017J01626).

\section{ACKNOWLEDGMENTS}

We would like to thank Editage (www.editage.com) for English language editing.

Ayoub, W. S., Steggerda, J., Yang, J. D., Kuo, A., Sundaram, V., and Lu, S. C. (2019). Current status of hepatocellular carcinoma detection: screening strategies and novel biomarkers. Ther. Adv. Med. Oncol. 11:1758835919869120. doi: 10.1177/ 1758835919869120

Boini, K. M., Xia, M., Abais, J. M., Li, G., Pitzer, A. L., Gehr, T. W., et al. (2014). Activation of inflammasomes in podocyte injury of mice on the high fat diet: effects of ASC gene deletion and silencing. Biochim. Biophys. Acta 1843, 836-845. doi: 10.1016/j.bbamcr.2014.01.033

Bortolotti, P., Faure, E., and Kipnis, E. (2018). Inflammasomes in tissue damages and immune disorders after trauma. Front. Immunol. 9:1900. doi: 10.3389/ fimmu.2018.01900

Bouattour, M., Raymond, E., Qin, S., Cheng, A. L., Stammberger, U., Locatelli, G., et al. (2018). Recent developments of c-Met as a therapeutic target in hepatocellular carcinoma. Hepatology 67, 1132-1149. doi: 10.1002/hep. 29496 
Cai, S. M., Yang, R. Q., Li, Y., Ning, Z. W., Zhang, L. L., Zhou, G. S., et al. (2016). Angiotensin-(1-7) improves liver fibrosis by regulating the NLRP3 inflammasome via redox balance modulation. Antioxid. Redox. Signal. 24, 795-812. doi: 10.1089/ars.2015.6498

Chen, Y., Pitzer, A. L., Li, X., Li, P. L., Wang, L., and Zhang, Y. (2015). Instigation of endothelial Nlrp3 inflammasome by adipokine visfatin promotes inter-endothelial junction disruption: role of HMGB1. J. Cell Mol. Med. 19, 2715-2727. doi: $10.1111 / \mathrm{jcmm} .12657$

Chen, Y., Wang, L., Pitzer, A. L., Li, X., Li, P. L., and Zhang, Y. (2016). Contribution of redox-dependent activation of endothelial Nlrp3 inflammasomes to hyperglycemia-induced endothelial dysfunction. J. Mol. Med. 94, 1335-1347. doi: 10.1007/s00109-016-1481-1485

Chitturi, S., and Farrell, G. C. (2001). Etiopathogenesis of nonalcoholic steatohepatitis. Semin. Liver Dis. 21, 27-41. doi: 10.1055/s-2001- 12927

El-Serag, H. B., and Kanwal, F. (2014). Epidemiology of hepatocellular carcinoma in the United States: where are we? Where do we go? Hepatology 60, 1767-1775. doi: $10.1002 /$ hep. 27222

Ershaid, N., Sharon, Y., Doron, H., Raz, Y., Shani, O., Cohen, N., et al. (2019). NLRP3 inflammasome in fibroblasts links tissue damage with inflammation in breast cancer progression and metastasis. Nat. Commun. 10:4375. doi: 10.1038/ s41467-019-12370-12378

Fan, J., Xie, K., Wang, L., Zheng, N., and Yu, X. (2019). Roles of inflammasomes in inflammatory kidney diseases. Mediators Inflamm. 2019:2923072. doi: 10.1155/ 2019/2923072

Fan, S. H., Wang, Y. Y., Lu, J., Zheng, Y. L., Wu, D. M., Li, M. Q., et al. (2014). Luteoloside suppresses proliferation and metastasis of hepatocellular carcinoma cells by inhibition of NLRP3 inflammasome. PLoS One 9:e89961. doi: 10.1371/ journal.pone.0089961

Forner, A., Llovet, J. M., and Bruix, J. (2012). Hepatocellular carcinoma. Lancet 379, 1245-1255. doi: 10.1016/S0140-6736(11)61347-61340

Guo, H., Bi, X., Zhou, P., Zhu, S., and Ding, W. (2017). NLRP3 deficiency attenuates renal fibrosis and ameliorates mitochondrial dysfunction in a mouse unilateral ureteral obstruction model of chronic kidney disease. Mediators Inflamm. 2017:8316560. doi: 10.1155/2017/8316560

Guo, Z., Yu, S., Chen, X., Ye, R., Zhu, W., and Liu, X. (2016). NLRP3 Is involved in ischemia/reperfusion injury. CNS Neurol. Disord. Drug Targets 15, 699-712. doi: $10.2174 / 1871527315666160321111829$

Han, C. Y., Rho, H. S., Kim, A., Kim, T. H., Jang, K., Jun, D. W., et al. (2018). FXR inhibits endoplasmic reticulum stress-induced NLRP3 inflammasome in hepatocytes and ameliorates liver injury. Cell Rep. 24, 2985-2999. doi: 10.1016/ j.celrep.2018.07.068

Henao-Mejia, J., Elinav, E., Jin, C., Hao, L., Mehal, W. Z., Strowig, T., et al. (2012). Inflammasome-mediated dysbiosis regulates progression of NAFLD and obesity. Nature 482, 179-185. doi: 10.1038/nature10809

Inchingolo, R., Posa, A., Mariappan, M., and Spiliopoulos, S. (2019). Locoregional treatments for hepatocellular carcinoma: current evidence and future directions. World J. Gastroenterol. 25, 4614-4628. doi: 10.3748/wjg.v25.i32. 4614

Inzaugarat, M. E., Johnson, C. D., Holtmann, T. M., McGeough, M. D., Trautwein, C., Papouchado, B. G., et al. (2019). NLR family pyrin domain-containing 3 inflammasome activation in hepatic stellate cells induces liver fibrosis in mice. Hepatology 69, 845-859. doi: 10.1002/hep.30252

Kelley, N., Jeltema, D., Duan, Y., and He, Y. (2019). The NLRP3 inflammasome: an overview of mechanisms of activation and regulation. Int. J. Mol. Sci. 20:3328. doi: 10.3390/ijms20133328

Koyama, Y., Xu, J., Liu, X., and Brenner, D. A. (2016). New developments on the treatment of liver fibrosis. Dig. Dis. 34, 589-596. doi: 10.1159/000445269

Lee, H. J., Yeon, J. E., Ko, E. J., Yoon, E. L., Suh, S. J., Kang, K., et al. (2015). Peroxisome proliferator-activated receptor-delta agonist ameliorated inflammasome activation in nonalcoholic fatty liver disease. World $\mathrm{J}$. Gastroenterol. 21, 12787-12799. doi: 10.3748/wjg.v21.i45.12787

Lee, Y. A., Wallace, M. C., and Friedman, S. L. (2015). Pathobiology of liver fibrosis: a translational success story. Gut 64, 830-841. doi: 10.1136/gutjnl-2014-306842

Lv, Y., Gao, X., Luo, Y., Fan, W., Shen, T., Ding, C., et al. (2019). Apigenin ameliorates HFD-induced NAFLD through regulation of the XO/NLRP3 pathways. J. Nutr. Biochem. 71, 110-121. doi: 10.1016/j.jnutbio.2019.05.015

Man, S. M., and Kanneganti, T. D. (2015). Regulation of inflammasome activation. Immunol. Rev. 265, 6-21. doi: 10.1111/imr.12296
Mangan, M. S. J., Olhava, E. J., Roush, W. R., Seidel, H. M., Glick, G. D., and Latz, E. (2018). Targeting the NLRP3 inflammasome in inflammatory diseases. Nat. Rev. Drug. Discov. 17, 588-606. doi: 10.1038/nrd.2018.97

Mathur, A., Hayward, J. A., and Man, S. M. (2018). Molecular mechanisms of inflammasome signaling. J. Leukoc. Biol. 103, 233-257. doi: 10.1189/jlb. 3MR0617-250R

Mitsuyoshi, H., Yasui, K., Hara, T., Taketani, H., Ishiba, H., Okajima, A., et al. (2017). Hepatic nucleotide binding oligomerization domain-like receptors pyrin domain-containing 3 inflammasomes are associated with the histologic severity of non-alcoholic fatty liver disease. Hepatol. Res. 47, 1459-1468. doi: 10.1111/hepr.12883

Mridha, A. R., Wree, A., Robertson, A. A. B., Yeh, M. M., Johnson, C. D., Van Rooyen, D. M., et al. (2017). NLRP3 inflammasome blockade reduces liver inflammation and fibrosis in experimental NASH in mice. J. Hepatol. 66, 1037-1046. doi: 10.1016/j.jhep.2017.01.022

Ning, Z. W., Luo, X. Y., Wang, G. Z., Li, Y., Pan, M. X., Yang, R. Q., et al. (2017). MicroRNA-21 mediates angiotensin II-Induced liver fibrosis by activating NLRP3 inflammasome/IL-1beta Axis via targeting Smad7 and Spry1. Antioxid. Redox. Signal. 27, 1-20. doi: 10.1089/ars.2016.6669

Sanchez-Valle, V., Chavez-Tapia, N. C., Uribe, M., and Mendez-Sanchez, N. (2012). Role of oxidative stress and molecular changes in liver fibrosis: a review. Curr. Med. Chem. 19, 4850-4860. doi: 10.2174/092986712803341520

Schuppan, D., Ashfaq-Khan, M., Yang, A. T., and Kim, Y. O. (2018). Liver fibrosis: direct antifibrotic agents and targeted therapies. Matrix. Biol. 6, 435-451. doi: 10.1016/j.matbio.2018.04.006

Schuppan, D., and Kim, Y. O. (2013). Evolving therapies for liver fibrosis. J. Clin. Invest. 123, 1887-1901. doi: 10.1172/JCI66028

Seki, E., and Brenner, D. A. (2015). Recent advancement of molecular mechanisms of liver fibrosis. J. Hepatobiliary Pancreat. Sci. 22, 512-518. doi: 10.1002/ jhbp. 245

Shi, C., Xue, W., Han, B., Yang, F., Yin, Y., and Hu, C. (2019). Acetaminophen aggravates fat accumulation in NAFLD by inhibiting autophagy via the AMPK/mTOR pathway. Eur. J. Pharmacol 850, 15-22. doi: 10.1016/j.ejphar. 2019.02.005

Strowig, T., Henao-Mejia, J., Elinav, E., and Flavell, R. (2012). Inflammasomes in health and disease. Nature 481, 278-286. doi: 10.1038/nature10759

Swanson, K. V., Deng, M., and Ting, J. P. (2019). The NLRP3 inflammasome: molecular activation and regulation to therapeutics. Nat. Rev. Immunol. 19, 477-489. doi: 10.1038/s41577-019-0165-160

Tan, W., Gu, Z., Leng, J., Zou, X., Chen, H., Min, F., et al. (2019). Let-7f$5 \mathrm{p}$ ameliorates inflammation by targeting NLRP3 in bone marrow-derived mesenchymal stem cells in patients with systemic lupus erythematosus. Biomed. Pharmacother. 118:109313. doi: 10.1016/j.biopha.2019.109313

van de Veerdonk, F. L., Netea, M. G., Dinarello, C. A., and Joosten, L. A. (2011). Inflammasome activation and IL-1beta and IL-18 processing during infection. Trends Immunol. 32, 110-116. doi: 10.1016/j.it.2011.01.003

Vonghia, L., Van Herck, M. A., Weyler, J., and Francque, S. (2019). Targeting myeloid-derived cells: new frontiers in the treatment of non-alcoholic and alcoholic liver disease. Front. Immunol. 10:563. doi: 10.3389/fimmu.2019.00563

Wan, L., Yuan, X., Liu, M., and Xue, B. (2018). miRNA-223-3p regulates NLRP3 to promote apoptosis and inhibit proliferation of hep3B cells. Exp. Ther. Med. 15, 2429-2435. doi: 10.3892/etm.2017.5667

Wan, X., Xu, C., Lin, Y., Lu, C., Li, D., Sang, J., et al. (2016a). Uric acid regulates hepatic steatosis and insulin resistance through the NLRP3 inflammasomedependent mechanism. J. Hepatol. 64, 925-932. doi: 10.1016/j.jhep.2015.11. 022

Wan, X., Xu, C., Yu, C., and Li, Y. (2016b). Role of NLRP3 Inflammasome in the Progression of NAFLD to NASH. Can. J. Gastroenterol. Hepatol. 2016:6489012. doi: 10.1155/2016/6489012

Wang, J. N., Li, L., Li, L. Y., Yan, Q., Li, J., and Xu, T. (2018). Emerging role and therapeutic implication of Wnt signaling pathways in liver fibrosis. Gene 674, 57-69. doi: 10.1016/j.gene.2018.06.053

Wang, L., Chen, Y., Li, X., Zhang, Y., Gulbins, E., and Zhang, Y. (2016). Enhancement of endothelial permeability by free fatty acid through lysosomal cathepsin B-mediated Nlrp3 inflammasome activation. Oncotarget 7, 7322973241. doi: 10.18632/oncotarget.12302

Wang, Y., Li, H., Li, Y., Zhao, Y., Xiong, F., Liu, Y., et al. (2019). Coriolus versicolor alleviates diabetic cardiomyopathy by inhibiting cardiac fibrosis and NLRP3 
inflammasome activation. Phytother. Res. 33, 2737-2748. doi: 10.1002/ptr. 6448

Watanabe, A., Sohail, M. A., Gomes, D. A., Hashmi, A., Nagata, J., Sutterwala, F. S., et al. (2009). Inflammasome-mediated regulation of hepatic stellate cells. Am. J. Physiol. Gastrointest. Liver Physiol. 296, G1248-G1257. doi: 10.1152/ajpgi. 90223.2008

Wei, Q., Guo, P., Mu, K., Zhang, Y., Zhao, W., Huai, W., et al. (2015). Estrogen suppresses hepatocellular carcinoma cells through ERbeta-mediated upregulation of the NLRP3 inflammasome. Lab Invest. 95, 804-816. doi: 10. 1038/labinvest.2015.63

Wei, Q., Mu, K., Li, T., Zhang, Y., Yang, Z., Jia, X., et al. (2014). Deregulation of the NLRP3 inflammasome in hepatic parenchymal cells during liver cancer progression. Lab Invest. 94, 52-62. doi: 10.1038/labinvest.2013.126

Wei, Q., Zhu, R., Zhu, J., Zhao, R., and Li, M. (2019). E2-Induced activation of the NLRP3 inflammasome triggers pyroptosis and inhibits autophagy in HCC Cells. Oncol. Res. 27, 827-834. doi: 10.3727/096504018X15462920753012

Wu, X., Zhang, F., Xiong, X., Lu, C., Lian, N., Lu, Y., et al. (2015). Tetramethylpyrazine reduces inflammation in liver fibrosis and inhibits inflammatory cytokine expression in hepatic stellate cells by modulating NLRP3 inflammasome pathway. IUBMB Life 67, 312-321. doi: 10.1002/ iub. 1348

Wu, X. Y., Li, K. T., Yang, H. X., Yang, B., Lu, X., Zhao, L. D., et al. (2019). Complement C1q synergizes with PTX3 in promoting NLRP3 inflammasome over-activation and pyroptosis in rheumatoid arthritis. J. Autoimmun. 106:102336. doi: 10.1016/j.jaut.2019.102336

Xiao, J., Liu, Y., Xing, F., Leung, T. M., Liong, E. C., and Tipoe, G. L. (2016). Bee's honey attenuates non-alcoholic steatohepatitis-induced hepatic injury through the regulation of thioredoxin-interacting protein-NLRP3 inflammasome pathway. Eur. J. Nutr. 55, 1465-1477. doi: 10.1007/s00394-0150964-964

Xiao, J., Wang, F., Liong, E. C., So, K. F., and Tipoe, G. L. (2018). ). Lycium barbarum polysaccharides improve hepatic injury through NFkappa-B and NLRP3/6 pathways in a methionine choline deficient diet steatohepatitis mouse model. Int. J. Biol. Macromol. 120(Pt B), 1480-1489. doi: 10.1016/j.ijbiomac. 2018.09.151

Yang, G., Lee, H. E., and Lee, J. Y. (2016). A pharmacological inhibitor of NLRP3 inflammasome prevents non-alcoholic fatty liver disease in a mouse model induced by high fat diet. Sci. Rep. 6:24399. doi: 10.1038/srep 24399

Yang, H., Huang, J., Gao, Y., Wen, Z., Peng, L., and Ci, X. (2019). Oridonin attenuates carrageenan-induced pleurisy via activation of the KEAP-1/Nrf2 pathway and inhibition of the TXNIP/NLRP3 and NF-kappaB pathway in mice. Inflammopharmacology. doi: 10.1007/s10787-019-00644-y [Epub ahead of print].

Yang, S. J., and Lim, Y. (2014). Resveratrol ameliorates hepatic metaflammation and inhibits NLRP3 inflammasome activation. Metabolism 63, 693-701. doi: 10.1016/j.metabol.2014.02.003
Yang, S., Yang, L., Li, X., Li, B., Li, Y., Zhang, X., et al. (2019). New insights into autophagy in hepatocellular carcinoma: mechanisms and therapeutic strategies. Am. J. Cancer Res 9, 1329-1353.

You, Y., Huang, Y., Wang, D., Li, Y., Wang, G., Jin, S., et al. (2019). Angiotensin (17) inhibits arecoline-induced migration and collagen synthesis in human oral myofibroblasts via inhibiting NLRP3 inflammasome activation. J. Cell Physiol. 234, 4668-4680. doi: 10.1002/jcp.27267

Zhang, Y., Chen, Y., Zhang, Y., Li, P. L., and Li, X. (2019). Contribution of cathepsin B-dependent Nlrp3 inflammasome activation to nicotine-induced endothelial barrier dysfunction. Eur. J. Pharmacol. 865:172795. doi: 10.1016/ j.ejphar.2019.172795

Zhang, Z., Wang, F., and Zhang, Y. (2019). Expression and contribution of nlrp3 inflammasome during the follicular development induced by PMSG. Front. Cell Dev. Biol. 7:256. doi: 10.3389/fcell.2019.00256

Zhang, Y., Li, X., Pitzer, A. L., Chen, Y., Wang, L., and Li, P. L. (2015). Coronary endothelial dysfunction induced by nucleotide oligomerization domain-like receptor protein with pyrin domain containing 3 inflammasome activation during hypercholesterolemia: beyond inflammation. Antioxid. Redox. Signal. 22, 1084-1096. doi: 10.1089/ars.2014.5978

Zheng, H., Wang, X., Zhang, Y., Chen, L., Hua, L., and Xu, W. (2019). Pien-TzeHuang ameliorates hepatic fibrosis via suppressing NF-kappaB pathway and promoting HSC apoptosis. J. Ethnopharmacol. 244:111856. doi: 10.1016/j.jep. 2019.111856

Zheng, T., Yang, X., Li, W., Wang, Q., Chen, L., Wu, D., et al. (2018). Salidroside attenuates high-fat diet-induced nonalcoholic fatty liver disease via AMPKDependent TXNIP/NLRP3 pathway. Oxid. Med. Cell Longev 2018:8597897. doi: $10.1155 / 2018 / 8597897$

Zhu, M. X., Wei, C. Y., Zhang, P. F., Gao, D. M., Chen, J., Zhao, Y., et al. (2019). Elevated TRIP13 drives the AKT/mTOR pathway to induce the progression of hepatocellular carcinoma via interacting with ACTN4. J. Exp. Clin. Cancer Res. 38:409. doi: 10.1186/s13046-019-1401-y

Zhu, W., Feng, P. P., He, K., Li, S. W., and Gong, J. P. (2018). Liraglutide protects non-alcoholic fatty liver disease via inhibiting NLRP3 inflammasome activation in a mouse model induced by high-fat diet. Biochem. Biophys. Res. Commun. 505, 523-529. doi: 10.1016/j.bbrc.2018. 09.134

Conflict of Interest: The authors declare that the research was conducted in the absence of any commercial or financial relationships that could be construed as a potential conflict of interest.

Copyright (C) 2020 Shi, Yang and Zhang. This is an open-access article distributed under the terms of the Creative Commons Attribution License (CC BY). The use, distribution or reproduction in other forums is permitted, provided the original author(s) and the copyright owner(s) are credited and that the original publication in this journal is cited, in accordance with accepted academic practice. No use, distribution or reproduction is permitted which does not comply with these terms. 